\title{
TWO RECENT DEVELOPMENTS CONCERNING THE MONTE CARLO METHOD
}

\author{
M. HÉNON \\ Observatoire de Nice, Nice, France
}

\begin{abstract}
This paper consists of two independent parts.
(1) The Monte Carlo method for computing the evolution of spherical stellar systems has been modified so that the computation can be continued after the time of formation of the central singularity. Results are presented for systems with equal and unequal star masses. The initial core-halo formation is followed by a general expansion of the cluster, while the central singularity absorbs a growing fraction of the total negative energy.

(2) Theoretical expressions of the 'diffusion coefficients', which describe the effect of encounters in a stellar system, contain a factor $\ln (\gamma N)$ where $N$ is the number of stars and $\gamma$ is a constant usually taken to be of the order of 0.4 . A reconsideration of the 'non-dominant terms' leads to a substantially lower value, of the order of 0.15 for equal masses and 0.075 for unequal masses with a typical distribution. This correction improves the agreement between $\mathrm{N}$-body and Monte Carlo simulations of spherical systems.
\end{abstract}

\section{Extension of Monte Carlo Models Beyond the Singularity}

All methods used so far to study the dynamical evolution of a spherical cluster under the effect of encounters indicate that the core of the system contracts, and that the contraction ends in a singular event at a finite time. When the system is described by a continuous distribution function, the central density becomes infinite at the critical time (Hénon, 1961; von Hoerner, 1968; Larson, 1970). In $N$-body simulations, a close binary forms near the centre (see reviews by Aarseth, 1973, 1975; Wielen, 1974, 1975). In Hénon's Monte Carlo models, the innermost shell collapses (Hénon, 1971). In the models of Spitzer and associates, the computation does not quite reach the critical time, but a small fraction of the mass near the centre appears to be headed for collapse (Spitzer and Thuan, 1972; Spitzer, 1975).

A theoretical explanation of this phenomenon has been given by Lynden-Bell and Wood (1968), who treated stars as molecules and applied thermodynamical concepts: the core of the system is 'hotter' than the outer regions, so that there is an outward flux of heat; the core loses energy, contracts, and becomes hotter as a consequence of the virial theorem. This process accelerates until the central singularity is formed.

What happens after the critical time? Unfortunately many methods fail at or before that time, because of technical difficulties related to the appearance of the singularity. The $N$-body simulations, however, are able to survive the event. They indicate that the central binary progressively shrinks and absorbs a growing fraction of the total negative energy of the system. An early analytical model (Hénon, 1961) also indicated that after the central singularity has formed, it will emit a continuous flow of energy.

Again the explanation of this behaviour is given by Lynden-Bell and Wood's 
mechanism, which does not stop when the singularity is formed: the central parts are still hotter than the outside, and there is still an outward heat flux. However, the system has exhausted its first source of energy, which was the contraction of the core. Therefore the energy must come now from the singularity itself. This will appear less mysterious if events are considered in more detail. Shortly before the critical time, only a small fraction of the core is rapidly contracting, and therefore feeding the energy flow; the rest of the system is in quasi-equilibrium on this short time scale and is only traversed by the energy flow, without contributing to it. The active region becomes smaller and smaller as the critical time is approached, and finally shrinks to a point. This is most clearly seen in Larson's (1970) figures, and was also predicted earlier (Hénon, 1961). Thus, at the critical time, the energy flux originates from the centre itself. Since no further contraction is possible, after the critical time the energy must continue to come from the central singularity.

In the $N$-body simulations with point masses, extracting energy from the singularity is no problem; the central binary can supply any amount of energy by shrinking. In Hénon's Monte Carlo method, as recently described (1973), the innermost shell begins to shrink at the critical time; it can also supply an unlimited amount of energy in this way, and thus it plays very much the same role as the central binary in the $N$-body simulations. Unfortunately, the results then become unreliable, for technical reasons: the method assumes that the shells which represent the system have closely spaced radii, approximating a continuous distribution; it does not function properly if a singular shell has a radius much smaller than the others. For this reason, the Monte Carlo computations have until now been stopped at the critical time.

On the other hand, simple estimates indicate that many real globular clusters, if not most, have already passed their critical time (Spitzer, 1975). Since the Monte Carlo method is especially designed for the case of globular clusters, it seems to be of prime importance to be able to extend it to the post-critical phase of evolution. We may note also that a recent comparison with $N$-body simulations for the case of unequal masses (Aarseth et al., 1975; also Wielen, 1975, Figure 2) was impeded by the early demise of the Monte Carlo models.

We present here an attempt to extend the Monte Carlo models past the critical time. The problem is to introduce at the centre a mechanism which can represent the singularity and act as an energy source, in a way which is physically reasonable and technically trouble-free. As already said, if the system is left to its own devices, it will use one shell for the purpose, and this creates technical difficulties. Therefore it was felt preferable to introduce at the centre an artificial energy source, entirely distinct from the shells. This source has no mass; its only role is to supply energy as required by the evolution of the cluster.

The detailed mechanism for the transfer of energy from the source to the rest of the system must now be specified. We take the view that the rate of flow of the energy is controlled by the system as a whole, not by the singularity. To justify this assumption, consider the near-real situation in $N$-body simulations, with mass points and a central binary. At any given time, the structure of the system corresponds to some 
definite temperature difference between the inner and outer parts, and therefore dictates a definite outward flux of energy. Suppose that the central singularity delivers too much energy with respect to this requirement. The excess energy will not be transmitted, but stored in the innermost fraction of the core, which will expand. This reduces the interaction between the central binary and the core, and the energy flux from the binary is brought down. Conversely, if the energy delivered is insufficient, the core contracts and the interaction with the binary increases. This regulating mechanism automatically adjusts the energy flux supplied by the binary to the amount required by the system. The situation is the same as in stellar interiors, where the rate of production of nuclear energy in the central region is automatically adjusted to the value required to maintain the overall equilibrium.

This has two consequences for our model. First, the physical nature of the energy source does not matter, and we shall in fact leave it unspecified. Second, the mechanism for the transfer of energy must be self-regulatory, as in the real case: the flux should increase when the innermost core contracts, and conversely, so as to bring about a stable state of affairs.

Let us define the self-energy of a shell as the energy which it would have in the absence of all other shells, i.e. (cf. Hénon, 1971):

$$
\frac{1}{2} K m\left(v_{r}^{2}+v_{t}^{2}\right)-\frac{1}{2} \frac{G K^{2} m^{2}}{r}
$$

where $r$ is the radius of the shell; $m, v_{r}, v_{t}$ are the mass, radial velocity and transverse velocity of each individual star in the shell, and $K$ is the number of stars in the shell. For a typical shell, the first term in (1) is of order $T / n$, where $T$ is the kinetic energy of the whole cluster and $n$ is the number of shells; the second term is of order $|W| / n^{2}$, where $W$ is the potential energy of the cluster. Thus the first term is usually much larger than the second, and the self-energy (1) is normally positive. On the other hand, experience with the previous models showed that when the innermost shell starts collapsing, its self-energy becomes negative. Physically, this means that the shell becomes an independent self-gravitating system, just as the binary in the $N$-body simulations.

Therefore the following simple procedure was introduced. The self-energy of the innermost shell is constantly monitored. Whenever it is found to be negative, it is brought back to a zero value; this is done by increasing the radial velocity $v_{r}$. The energy used in this operation is considered to have been given by the central energy source.

This simple dẹvice was found to work quite satisfactorily. Figure 1 represents three 'old' Monte Carlo computations (thin lines), already shown by Wielen (1975, Figure 1), and a new computation (thick line), in which a central energy source has been introduced as explained above. The initial conditions are of course identical in all cases. All stars have the same mass. The old models stop at about $t=1.5$ because of the collapse of the innermost shell. In the new model, this collapse does not happen any more, and the computation can be continued without difficulty past the critical 


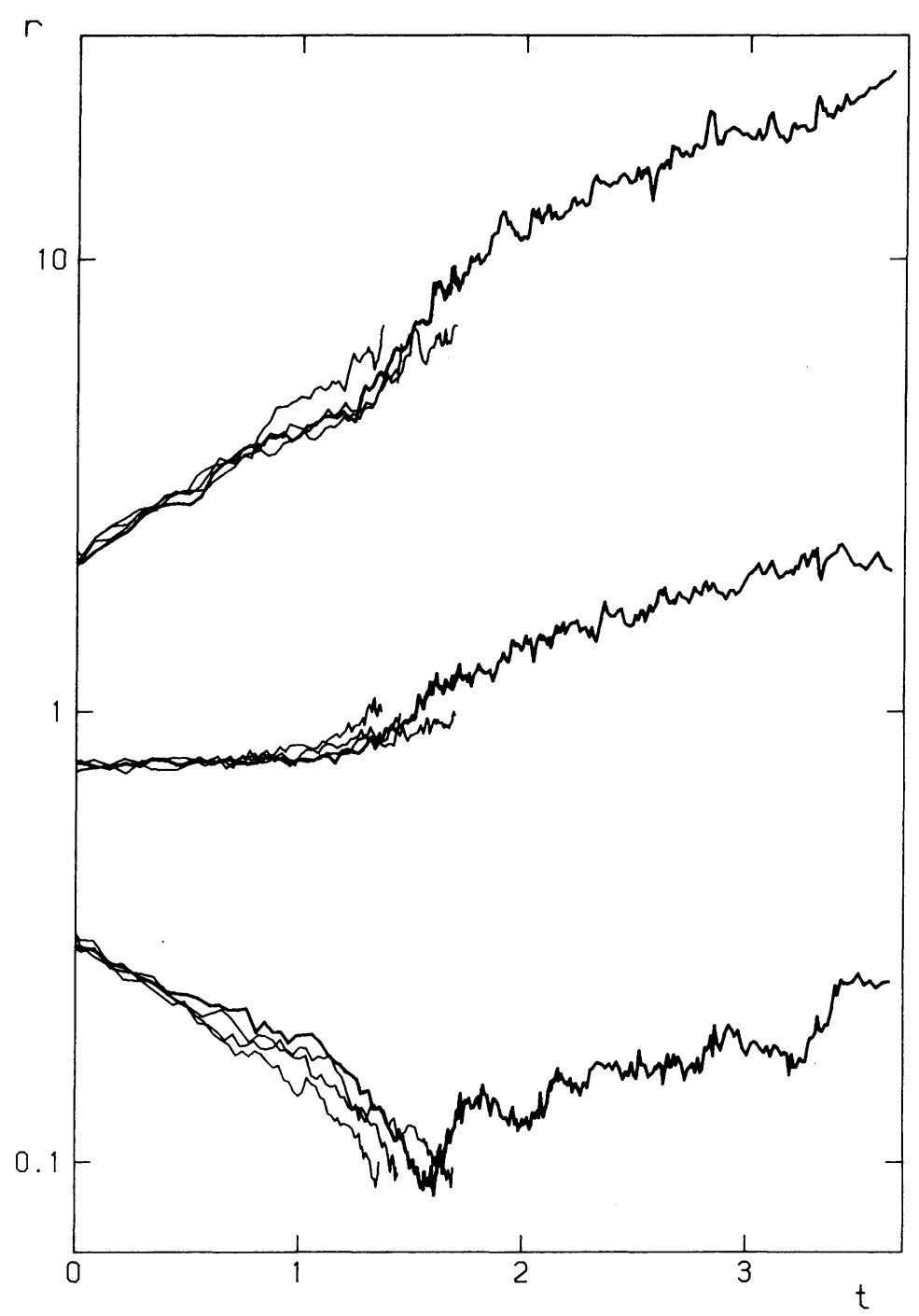

Fig. 1. Comparison of three old Monte Carlo models (thin lines) and one new model (thick line) which goes beyond the singularity. The curves represent the radii containing $10 \%, 50 \%, 90 \%$ of the mass, vs time. All stars have the same mass. The initial state is Plummer's model with isotropic velocity distribution.

time and for as long as desired. Figure 2 represents the total energy supplied by the central source since the beginning, as a function of time; the slope of the curve is the energy flux. It should be noted that the energy source is not just turned on at the critical time, but is present from the beginning. However, Figure 2 shows that the energy supplied by the source before the critical time is negligible. Therefore the source has no effect on the evolution during the first phase, and indeed Figure 1 shows that there is no significant difference between the new and the old models. 
As the critical time is approached, however, the energy flux begins to increase sharply (Figure 2), while the contraction of the core is halted and even reversed (Figure 1).

Thus, the evolution appears to consist of two rather different phases. The first phase, which lasts until the critical time at about $t=1.5$, is characterized by the usual core-halo formation. In the second phase, after the critical time, we observe a general

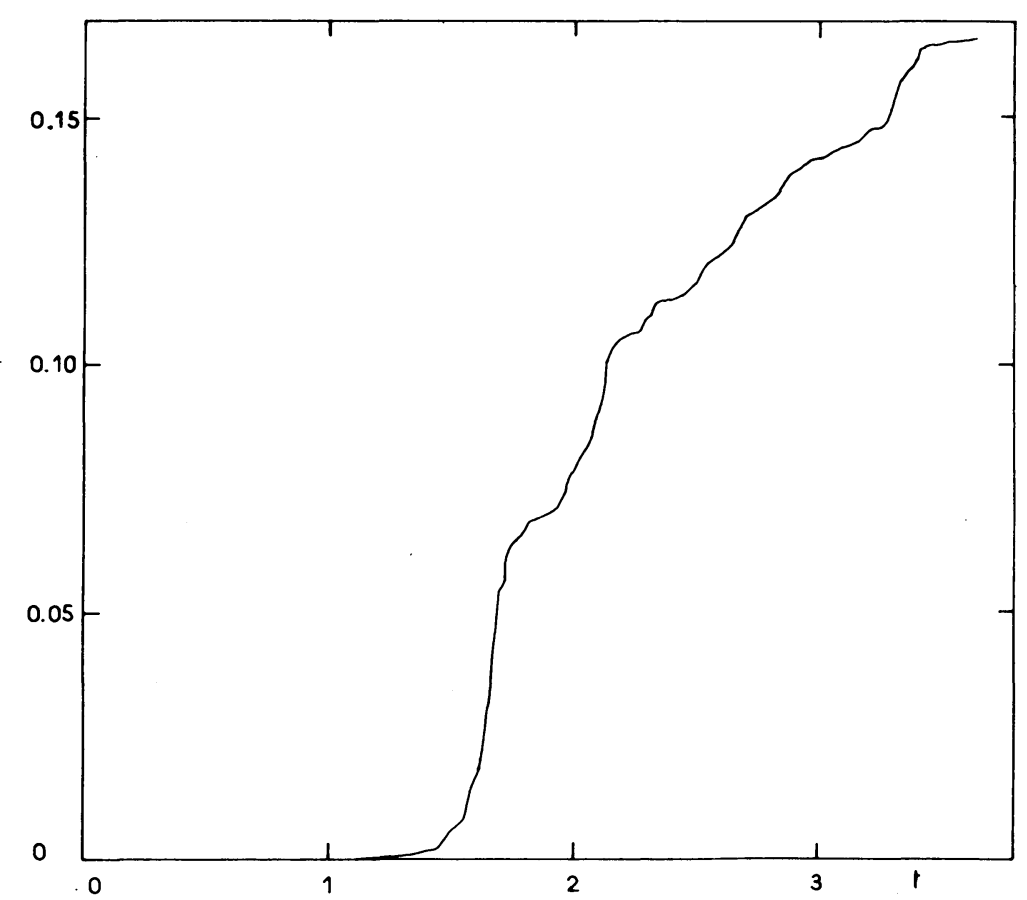

Fig. 2. Energy delivered by the central source, as a function of time, for the new model of Figure 1.

expansion of the system; this is of course made possible by the fact that the system now receives energy from the central source. The initial total energy of the system is -0.25 in our units; Figure 2 shows that at the end of the computation, a negative energy equal to about -0.165 has been absorbed in the central singularity, so that the rest of the system is left with about $\frac{1}{3}$ of its initial negative energy. Further evolution will in all probability follow the same trend, with the cluster expanding indefinitely while more and more of the total negative energy is absorbed by the central source. The evolution slows down with time, because the relaxation time increases as the cluster expands. Therefore the curve of Figure 2 should asymptotically approach the limiting value 0.25 .

This description of the final evolution applies only to the present idealized case of an isolated cluster. In real clusters, tidal effects will certainly affect the evolution, and probably bring about a full dissolution of the system after a finite time (Wielen, 1971).

Figure 1 shows also that the halo expands faster than the core. This, unfortunately, 
seems to rule out the use of a simple homological model for describing the final phase of the evolution.

Figure 3 again represents three old Monte Carlo models (thin lines), already shown by Wielen (1975, Figure 2), and a new model (thick line), for a case with un-

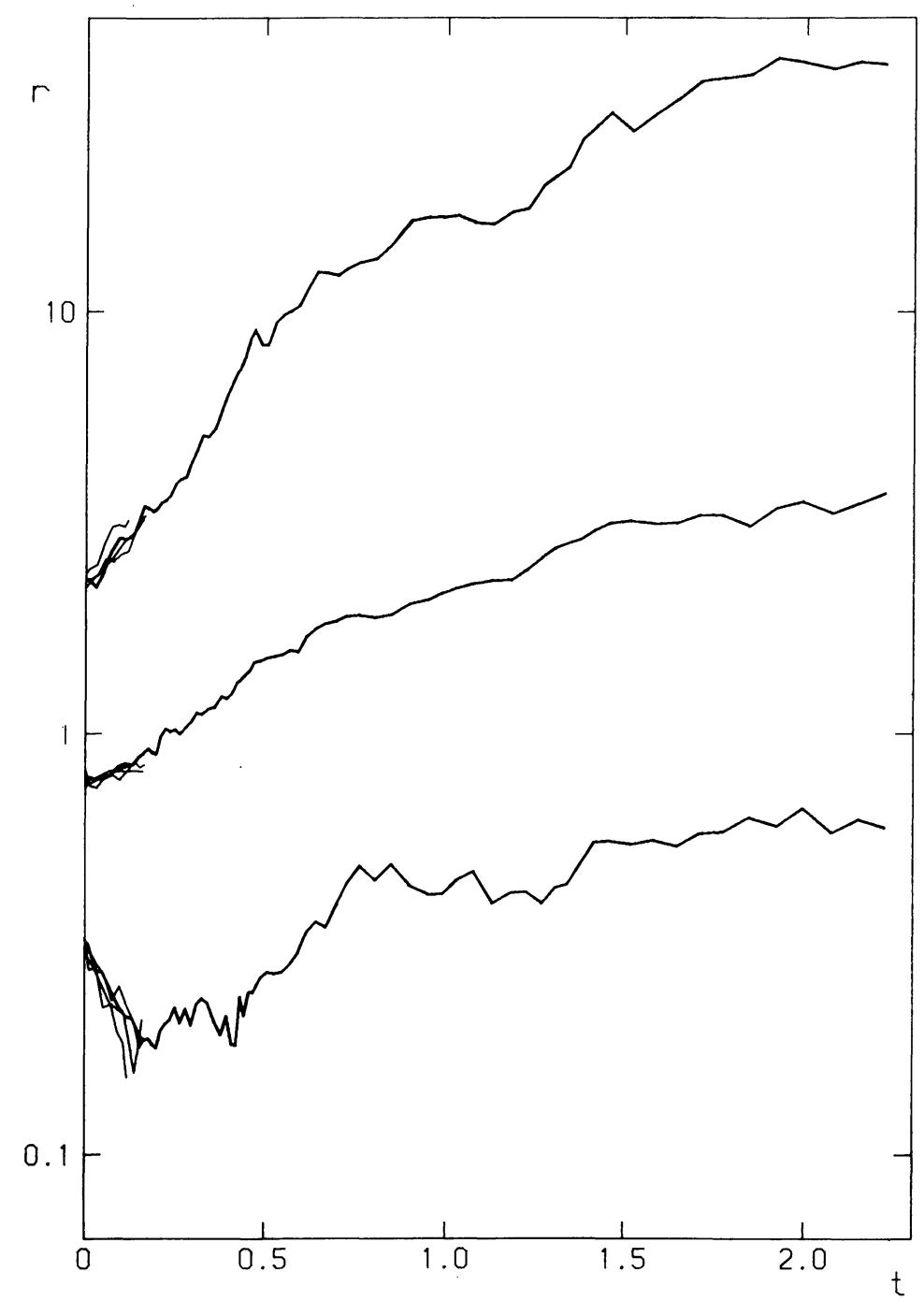

Fig. 3. Comparison of three old Monte Carlo models (thin lines) and one new model (thick line) which goes beyond the singularity. The curves have the same meaning as in Figure 1 . The masses of the stars are distributed according to Wielen's law. The initial state is the same as in Figure 1.

equal masses. Here the progress is even more apparent: the old models are stopped in the vicinity of $t=0.15$, while the new model has been computed beyond $t=2$ and could be easily extended still further. Figure 4 represents the growth of the total energy supplied by the central source. These two figures are qualitatively similar to 


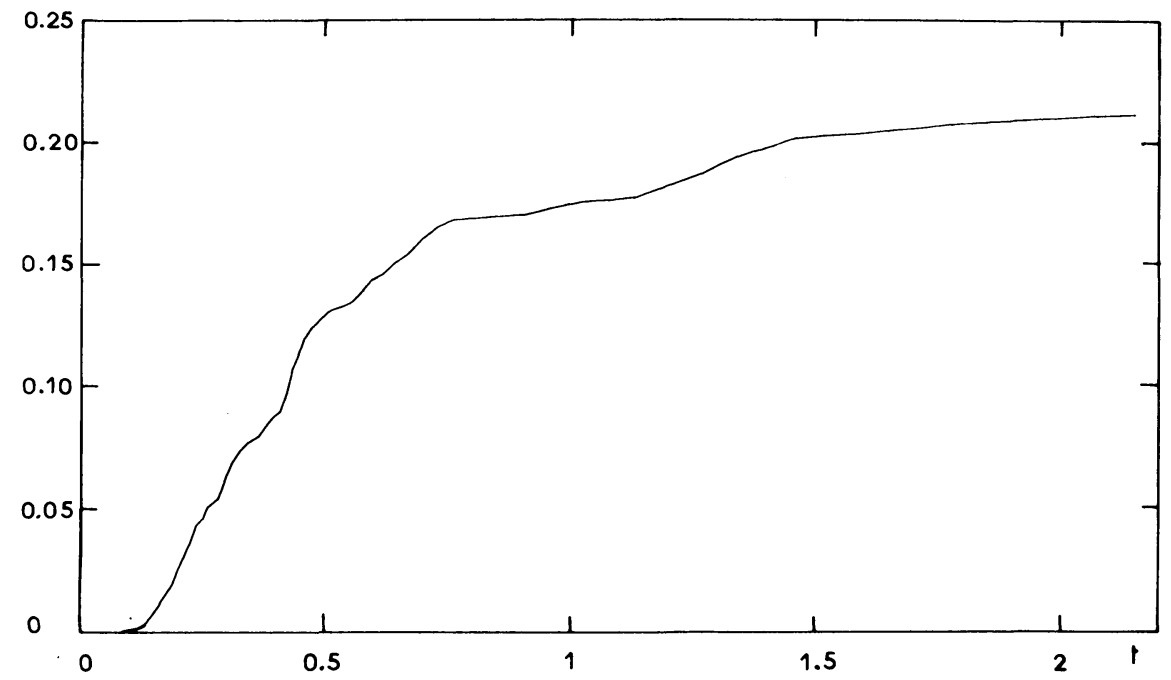

Fig. 4. Energy delivered by the central source, as a function of time, for the new model of Figure 3.

Figures 1 and 2: again there is a first phase of core-halo formation, terminating at $t \approx 0.15$, and a second phase of general expansion; and the central source absorbs a growing fraction of the total negative energy. At the end of the computation, this fraction is about $85 \%$. The asymptotic approach of the limiting value 0.25 is apparent on Figure 4. This figure is also in qualitative agreement with the results obtained by Aarseth (1971) from $N$-body simulations.

\section{Non-Dominant Terms in the Diffusion Coefficients}

The Monte Carlo method (Hénon, 1971, 1973) relies on the classical theory of relaxation due to two-body encounters. This theory involves a number of approximations. Until recently, there was no pressing need to introduce refinements, since no accurate data were available against which the theoretical results could be tested; observational evidence has been particularly disappointing in that respect (cf. King, 1975). Today, however, the situation is changing; $N$-body simulations provide a wealth of very detailed information, against which the theory can be matched (Wielen, 1975). There is also the hope that new observational techniques will improve our knowledge of real clusters, for instance by allowing a reliable determination of individual velocities. Therefore it may be worthwhile to reconsider some of the approximations in the theory.

One of the standard approximations consists in neglecting the 'non-dominant terms' (Chandrasekhar, 1941), i.e. the terms which are of order $1 / \ln N$ with respect to the dominant terms. $N$ is the number of stars in the system. This approximation is clearly justified in the limit of $N$ very large. For $N$ of the order of 100 to 500 , however, the factor $1 / \ln N$ is not so small and non-dominant terms could have a significant effect. 
Consider a test star with mass $m_{1}$ and velocity $\mathbf{V}_{1}$. Its change of velocity as a result of two-body encounters is governed by the 'diffusion coefficients', i.e. the components of $\left\langle\Delta \mathbf{V}_{1}\right\rangle,\left\langle\Delta \mathbf{V}_{1} \Delta \mathbf{V}_{1}\right\rangle$, etc. For simplicity, we shall consider here only one scalar coefficient: $\left\langle\left(\Delta \mathbf{V}_{1}\right)^{2}\right\rangle$, the mean square of the change of the velocity vector (not to be confused with the change in absolute velocity). This quantity may be considered as typical since it incorporates both radial and transversal diffusion. It is given by a comparatively simple expression (cf. Hénon, 1973):

$$
\begin{aligned}
\left\langle\left(\Delta \mathbf{V}_{1}\right)^{2}\right\rangle=4 \pi G^{2} \Delta t \iiint \int f & \left(\mathbf{V}_{2}, m_{2}\right) m_{2}^{2} V^{-1} \times \\
& \times \ln \left\{1+\left[\frac{l_{\max } V^{2}}{G\left(m_{1}+m_{2}\right)}\right]^{2}\right\} \mathrm{d} \mathbf{V}_{2} \mathrm{~d} m_{2} .
\end{aligned}
$$

Here $\Delta t$ is the interval of time considered; $f\left(\mathbf{V}_{2}, m_{2}\right)$ is the distribution function, defined as number of points per unit volume in a seven-dimensional phase space $\left(\mathbf{r}_{2}, \mathbf{V}_{2}, m_{2}\right) ; V=\left|\mathbf{V}_{2}-\mathbf{V}_{1}\right|$ is the relative velocity of the two encountering stars; $l_{\max }$ is the maximum impact distance. Formula (2) is exact, within the frame of the two-body encounter theory: no approximations have been made so far.

$l_{\max }$ is of the order of the dimensions of the system; we shall adopt here the value recommended by Spitzer and Hart (1971):

$$
l_{\max }=R_{h},
$$

where $R_{h}$ is the radius containing half the mass, itself given in good approximation by (Spitzer, 1969):

$$
R_{h}=0.4 G M /\left\langle V_{1}^{2}\right\rangle,
$$

where $M$ is the mass of the system and $\left\langle V_{1}^{2}\right\rangle$ is the mean square velocity.

The argument of the logarithm in (2) is large: if we replace $V^{2}$ by its mean value, $2\left\langle V_{1}^{2}\right\rangle$ (since $V$ is the relative velocity between two stars), and also $m_{1}$ and $m_{2}$ by their mean value, $\langle m\rangle=M / N$, this argument becomes:

$$
1+(0.4 N)^{2} \text {. }
$$

Therefore the term 1 can clearly be neglected.

From this point, the classical treatment continues by noting that since this large quantity is the argument of a logarithm, its exact value does not matter very much; therefore it is actually replaced by $(0.4 N)^{2}$. Then the logarithm becomes a constant which can conveniently be taken out of the integrations. Here, we shall instead keep the exact expression (2), omitting only the constant 1 . After substitution of (3) and (4), we write it in the form

$$
\begin{aligned}
& \left\langle\left(\Delta \mathbf{V}_{1}\right)^{2}\right\rangle=8 \pi G^{2} \Delta t \iiint \int_{\int} f\left(\mathbf{V}_{2}, m_{2}\right) m_{2}^{2} V^{-1} \times \\
& \left.\quad \times\left\{\ln (0.4 N)+\ln \left(V^{2} / 2\left\langle V_{1}^{2}\right\rangle\right)+\ln \left[2\langle m\rangle / m_{1}+m_{2}\right)\right]\right\} \mathrm{d} \mathbf{V}_{2} \mathrm{~d} m_{2}
\end{aligned}
$$


which clearly separates the dominant term (first term in braces) from the nondominant terms (second and third terms). The classical approximation consists in omitting these non-dominant terms.

The diffusion coefficient (6) depends on the velocity $V_{1}$ and mass $m_{1}$ of the test star. In order to obtain a mean value for the effect of the non-dominant terms, we consider now the average of (6) over all test stars. In so doing, it seems physically most reasonable to weigh each star by its mass, so that we compute in effect

$$
\left\langle\left\langle\left(\Delta \mathbf{V}_{1}\right)^{2}\right\rangle\right\rangle=\frac{\iiint \int\left\langle\left(\Delta \mathbf{V}_{1}\right)^{2}\right\rangle f\left(\mathbf{V}_{1}, m_{1}\right) \mathrm{d} \mathbf{V}_{1} m_{1} \mathrm{~d} m_{1}}{\iiint \int f\left(\mathbf{V}_{1}, m_{1}\right) \mathrm{d} \mathbf{V}_{1} m_{1} \mathrm{~d} m_{1}}
$$

The denominator is then simply the local density $\varrho$. We have

$$
\left\langle\left\langle\left(\Delta \mathbf{V}_{1}\right)^{2}\right\rangle\right\rangle=8 \pi G^{2} \varrho^{-1} \Delta t\left[I_{1} \ln (0.4 N)+I_{2}+I_{3}\right],
$$

with

$$
\begin{aligned}
& I_{1}=\int_{8} f_{1} f_{2} m_{1} m_{2}^{2} V^{-1} \mathrm{~d} \mathbf{V}_{1} \mathrm{~d} \mathbf{V}_{2} \mathrm{~d} m_{1} \mathrm{~d} m_{2}, \\
& I_{2}=\int_{8} f_{1} f_{2} m_{1} m_{2}^{2} V^{-1} \ln \left(V^{2} / 2\left\langle V_{1}^{2}\right\rangle\right) \mathrm{d} \mathbf{V}_{1} \mathrm{~d} \mathbf{V}_{2} \mathrm{~d} m_{1} \mathrm{~d} m_{2}, \\
& I_{3}=\int_{8} f_{1} f_{2} m_{1} m_{2}^{2} V^{-1} \ln \left[2\langle m\rangle /\left(m_{1}+m_{2}\right)\right] \mathrm{d} \mathbf{V}_{1} \mathrm{~d} \mathbf{V}_{2} \mathrm{~d} m_{1} \mathrm{~d} m_{2}, \\
& f_{1}=f\left(\mathbf{V}_{1}, m_{1}\right), \quad f_{2}=f\left(\mathbf{V}_{2}, m_{2}\right) .
\end{aligned}
$$

(8) can also be written

$$
\left\langle\left\langle\left(\Delta \mathbf{V}_{1}\right)^{2}\right\rangle\right\rangle=8 \pi G^{2} \varrho^{-1} \Delta t I_{1} \ln (\gamma N),
$$

with

$$
\ln \gamma=\ln (0.4)+I_{2} / I_{1}+I_{3} / I_{1} .
$$

Thus the effect of the non-dominant terms will be simply to modify the dimensionless constant $\gamma$ in (10).

We consider first the case of equal masses:

$$
f\left(\mathbf{V}_{1}, m_{1}\right)=g\left(\mathbf{V}_{1}\right) \delta\left(m_{1}-m_{0}\right) .
$$

Then $I_{3}=0$, and

$$
\frac{I_{2}}{I_{1}}=\frac{\int_{6} g\left(\mathbf{V}_{1}\right) g\left(\mathbf{V}_{2}\right) V^{-1} \ln \left(V^{2} / 2\left\langle V_{1}^{2}\right\rangle\right) \mathrm{d} \mathbf{V}_{1} \mathrm{~d} \mathbf{V}_{2}}{\int_{6} g\left(\mathbf{V}_{1}\right) g\left(\mathbf{V}_{2}\right) V^{-1} \mathrm{~d}_{1} \mathrm{~d} \mathbf{V}_{2}} .
$$

We restrict ourselves now to isotropic velocity distributions, i.e. $g\left(\mathbf{V}_{1}\right)$ will depend only on the velocity modulus $V_{1}$. The integrals in (13) can be evaluated exactly for some particular forms of $g$. For a Maxwellian distribution,

$$
g\left(V_{1}\right)=a \exp \left(-j^{2} V_{1}^{2}\right)
$$


with $a, j$ constants, the result is

$$
I_{2} / I_{1}=\ln \left(\frac{2}{3}\right)-C=-0.9827 \ldots, \quad \gamma=0.1497 \ldots
$$

where $C=0.5772 \ldots$ is Euler's constant. For a truncated power law,

$$
g\left(V_{1}\right)=a V_{1}^{n} \text { for } V_{1}<V_{0}, \quad g\left(V_{1}\right)=0 \text { for } V_{1}>V_{0},
$$

with, $a, V_{0}$ constants and $n$ an integer, we find for $n$ even, $n \geqslant 0$ :

$$
\begin{aligned}
\frac{I_{2}}{I_{1}}=\ln \frac{2(n+5)}{n+3} & -2-\frac{2}{n+3}-\frac{2}{2 n+5}+ \\
+ & \frac{2}{n+2}\left(1+\frac{1}{3}+\frac{1}{5}+\cdots+\frac{1}{n+1}\right),
\end{aligned}
$$

and for $n$ odd, $n \geqslant-1$ :

$$
\begin{aligned}
\frac{I_{2}}{I_{1}}=\ln \frac{2(n+5)}{n+3} & -2-\frac{2}{n+3}-\frac{2}{2 n+5}+ \\
+ & \frac{2}{n+2}\left(\ln 2+\frac{1}{2}+\frac{1}{4}+\frac{1}{6}+\cdots+\frac{1}{n+1}\right) .
\end{aligned}
$$

These expressions are evaluated for a few values of $n$ in Table I. The last line, $n=\infty$,

TABLE I

\begin{tabular}{rcc}
\multicolumn{3}{c}{$\begin{array}{c}\text { Values of } \gamma \text { for a velocity distribution of } \\
\text { the form (16) }\end{array}$} \\
\hline$n$ & $I_{2} / I_{1}$ & $\gamma$ \\
\hline-1 & -0.8941 & 0.1636 \\
0 & -0.8627 & 0.1688 \\
1 & -0.8917 & 0.1640 \\
2 & -0.9259 & 0.1585 \\
3 & -0.9571 & 0.1536 \\
4 & -0.9840 & 0.1495 \\
$\ldots$ & -1.3069 & \\
$\infty$ & & 0.1083
\end{tabular}

corresponds to a $\delta$-distribution where all stars have the same velocity modulus: $g \propto \delta\left(V_{1}-V_{0}\right)$.

We note that $I_{2} / I_{1}$ is always negative. This can be explained by the presence of a factor $V^{-1}$ in the integrals, which gives more weight to low values of $V$. In physical terms: encounters with a low relative velocity are more effective. As a result, the true mean value of the logarithm is lower than the value derived in the classical treatment, where $V^{2}$ is simply replaced by its average.

We note also from (15) and Table I that, if one excepts the rather unphysical case of equal velocity moduli $(n=\infty$ in Table I), the value of $\gamma$ appears to be not very sensitive to the shape of the distribution function. This fortunate fact suggests that 
one can, with very little error, adopt a standard value for $\gamma$. We shall adopt here the value (15) corresponding to a Maxwellian distribution of velocities, rounded to

$$
\gamma=0.15 \text {. }
$$

Our results should be compared with an earlier computation of the effect of the non-dominant terms by Chandrasekhar (1941). A direct comparison is not possible, because Chandrasekhar considers a different quantity: $\left\langle(\Delta E)^{2}\right\rangle$, where $\Delta E$ is the energy exchanged between the two stars during an encounter. In our notations, there is

$$
\Delta E=\Delta\left(\frac{1}{2} m_{1} \mathbf{V}_{1}^{2}\right)=m_{1}\left[\mathrm{~V}_{1} \Delta \mathrm{V}_{1}+\frac{1}{2}\left(\Delta \mathrm{V}_{1}\right)^{2}\right]
$$

so that $\left\langle(\Delta E)^{2}\right\rangle$ actually involves diffusion coefficients up to the fourth order. One can, however, compute for this quantity the correction for non-dominant terms exactly as we did for $\left\langle\left(\Delta \mathbf{V}_{1}\right)^{2}\right\rangle$. We consider again the equal-mass case. For field stars with a maxwellian distribution of velocities, and for a test star of given velocity, $\left\langle(\Delta E)^{2}\right\rangle$ is given by Equation (81) in Chandrasekhar (1941). We define a mean value $\left\langle\left\langle(\Delta E)^{2}\right\rangle\right\rangle$ over all test stars. Here again it is found to be of the form

$$
\left\langle\left\langle(\Delta E)^{2}\right\rangle\right\rangle \propto I_{1} \ln (0.4 N)+I_{2}=I_{1} \ln (\gamma N),
$$

as in (8) and (10). The integrals $I_{1}$ and $I_{2}$ can be evaluated numerically, using Equation (81) and Tables 2 and 4 in Chandrasekhar (1941). The values of $g$ for $x_{0}<0.6$, not given by Chandrasekhar, have been taken equal to zero. The maximum impact distance, $l_{\max }$, is erroneously identified by Chandrasekhar with the average distance between neighbour stars, $D_{0}$; therefore $D_{0}$ should be replaced in his formulas by $l_{\max }$, itself given by (3) and (4). The result is then

$$
I_{2} / I_{1}=-1.0046, \quad \gamma=0.1465,
$$

which agrees rather well with (19). This indicates that $\gamma$ is not very sensitive to the particular diffusion coefficient, or combination of them, which is considered.

Liboff (1959) computed the transport coefficients in a fully ionized plasma, with a Maxwellian distribution of velocities. Although his emphasis was on a more correct treatment of the long-distance cutoff, he also computed exactly the effect of the nondominant terms for short distances. Our quantity $\left\langle\left\langle\left(\Delta \mathbf{V}_{1}\right)^{2}\right\rangle\right\rangle$ can be expressed in terms of Liboff's integral $\Omega_{1, c}^{(1)}$, and our result (15) can be shown to agree with his Equation (4.28).

Figure 5 shows again the four Monte Carlo models of Figure 1 (full lines), together with the results of $N$-body simulations by Aarseth (1974) and Wielen (1974) (filled and open symbols). The value $\gamma=0.15$ is used. This figure should be compared with Aarseth et al. (1975, Figure 1) or Wielen (1975, Figure 1) where the same data are represented, but the classical value $\gamma=0.4$ is used. It will be seen that the agreement is significantly improved with the new value of $\gamma$. There is, in fact, no noticeable deviation any more between the results of the two methods in Figure 5, except perhaps in the lower right, where the radius corresponding to $10 \%$ of the mass appears 


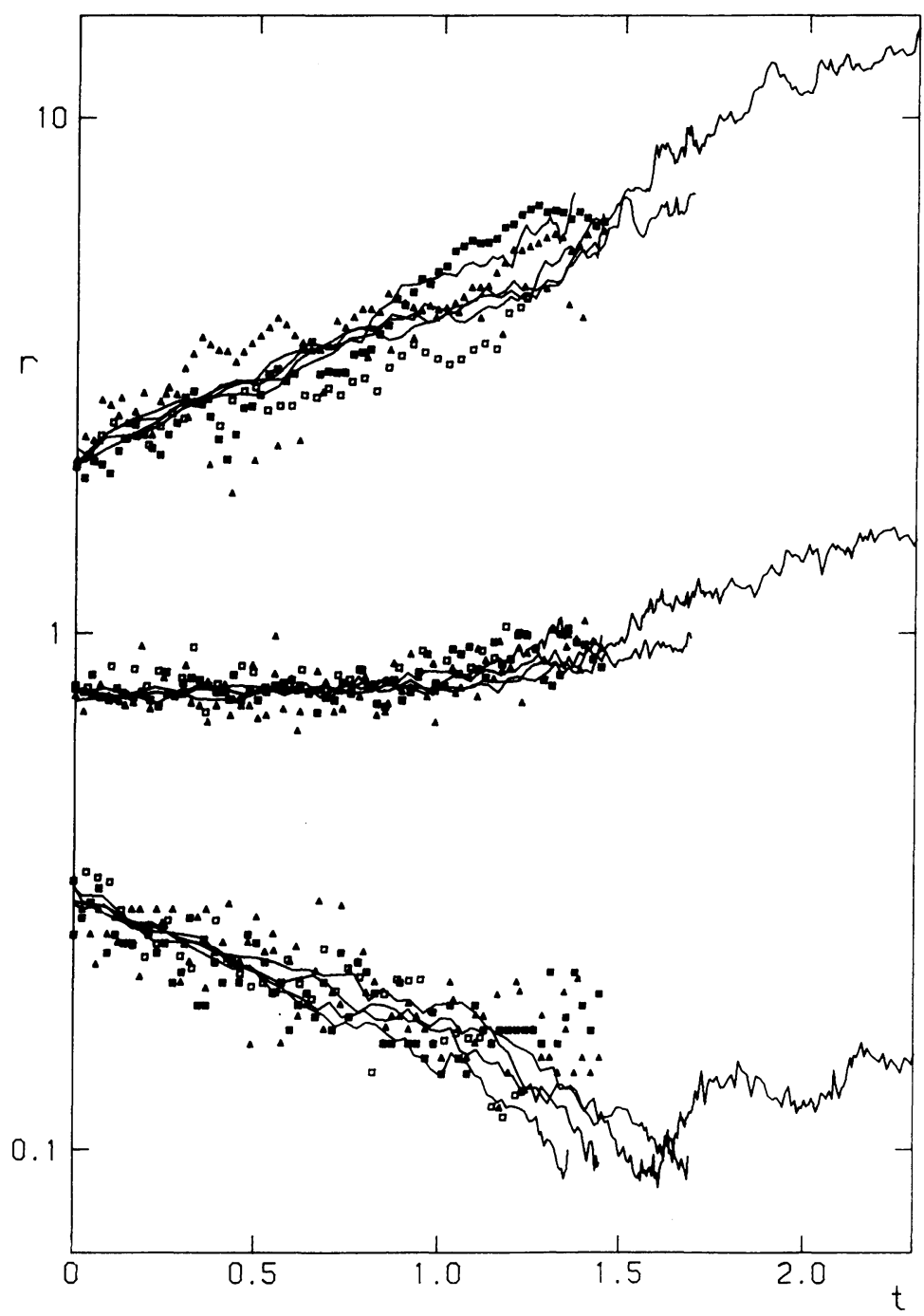

Fig. 5. Comparison of four Monte Carlo models (full lines) with four $N$-body simulations (symbols) for the equal-mass case, using the new value $\gamma=0.15$. The data represent the radii containing $10 \%, 50 \%, 90 \%$ of the mass, vs time. The Monte Carlo models are the same as in Figure 1. Filled triangles and squares represent two $N$-body simulations by Aarseth, with $N=250$. Open triangles and squares represent two $N$-body simulations by Wielen, with $N=100$ and $N=250$ respectively. The initial state is Plummer's model with isotropic velocity distribution.

to reach a minimum at a somewhat earlier time and at a higher value in the $N$-body simulations than in the Monte Carlo models.

A change in the value of $\gamma$ affects not merely the comparison between Monte Carlo and $N$-body results, but also the internal comparison between $N$-body simulations for different values of $N$; for, in order to do such a comparison, the simulations must be reduced to a common evolutionary scale, and this can be done only by borrowing 
from the theory the law of dependence of the evolution rate on $N$. In the present paper, the unit of time for each model was taken as (Aarseth et al., 1974):

$$
t_{0}=G M^{5 / 2}(-4 \mathscr{E})^{-3 / 2} N / \ln (\gamma N),
$$

where $\mathscr{E}$ is the total energy of the system; $t_{0}$ is the theoretical relaxation time, apart from a dimensionless multiplicative constant. As a result, when $\gamma$ is replaced by a different value $\gamma^{\prime}$, the apparent rate of evolution of a $N$-body simulation is multiplied by

$$
\ln (\gamma N) / \ln \left(\gamma^{\prime} N\right)
$$

This factor depends on $N$ : for $\gamma=0.4$ and $\gamma^{\prime}=0.15$, its value is 1.3622 and 1.2706 respectively for $N=100$ and 250 , which are the values used in the $N$-body simulations of Figure 5. On the other hand, the Monte Carlo results correspond to the limit $N \rightarrow \infty$, and are not affected by a change in $\gamma$ : the factor (24) reduces to 1 in this limit.

We consider now the case of unequal masses. We shall assume for simplicity that the distribution function is separable, i.e. there is no mass segregation:

$$
f\left(\mathbf{V}_{1}, m_{1}\right)=g\left(\mathbf{V}_{1}\right) h\left(m_{1}\right)
$$

Then $I_{2} / I_{1}$ has the same expression (13) as before, depending only on the velocity distribution $g$. On the other hand, we must now compute the third term in (11), which is no longer zero:

$$
\frac{I_{3}}{I_{1}}=\frac{\iint h\left(m_{1}\right) h\left(m_{2}\right) m_{1} m_{2}^{2} \ln \left[2\langle m\rangle /\left(m_{1}+m_{2}\right)\right] \mathrm{d} m_{1} \mathrm{~d} m_{2}}{\int h\left(m_{1}\right) m_{1} \mathrm{~d} m_{1} \int h\left(m_{2}\right) m_{2}^{2} \mathrm{~d} m_{2}} .
$$

$\langle m\rangle$ is given by

$$
\langle m\rangle=M / N=\int h(m) m \mathrm{~d} m / \int h(m) \mathrm{d} m .
$$

The corrective term (26) depends only on the mass distribution $h$. We evaluate it first for a continuous distribution:

$$
h(m)=a m^{-2} \text { for } m_{0}<m<Q m_{0}, \quad h(m)=0 \text { elsewhere, }
$$

with $a, m_{0}, Q$ constants. We obtain

$$
\begin{aligned}
\frac{I_{3}}{I_{1}}= & \ln \left(\frac{2 Q \ln Q}{Q-1}\right)-\frac{Q \ln Q}{Q-1}- \\
& -\frac{D(1+Q)+Q D(1+1 / Q)+(1+Q) \ln \left[4 Q /(1+Q)^{2}\right]+(1+Q) \pi^{2} / 12}{(Q-1) \ln Q}
\end{aligned}
$$


where $D$ is the dilogarithm function (Abramowitz and Stegun, 1965):

$$
D(x)=-\int_{1}^{x} \frac{\ln t}{t-1} \mathrm{~d} t .
$$

The expression (29) has been tabulated for a few values of $Q$ in Table II.

TABLE II

Values of the corrective term

$I_{3} / I_{1}$ for a mass distribution

of the form (28)

\begin{aligned} & \hline$Q I_{3} / I_{1} \\ &$\hline 2-0.0498 \\ & 4-0.1964 \\ & 8-0.4322 \\ & 16-0.7458 \\ & 32-1.1246 \\ & 64-1.5562 \\ & \hline\end{aligned}

We consider next the case of a discrete distribution:

$$
h(m)=a \sum_{i=1}^{k} n_{i} \delta\left(m-m_{i}\right)
$$

and the frequently used law for the masses $m_{i}$ and the corresponding numbers $n_{i}$ :

$$
m_{i}=2^{i-1}, \quad n_{i}=2^{k-i} .
$$

The integrals in (26) are replaced by simple sums. Results are given in Table III.

\section{TABLE III}

\begin{tabular}{l}
$\begin{array}{c}\text { Values of the corrective term } \\
I_{3} / I_{1} \text { for a mass distribution } \\
\text { of the form }(31,32)\end{array}$ \\
\hline$k$
\end{tabular}

These results correspond closely to those of Table II for $Q=2^{k}$, since (32) is the discrete equivalent of (28).

Finally, for Wielen's (1967) discrete distribution, which corresponds to $k=4$; 
$m_{i}=1,2,4,8 ; n_{i}=62,24,10,4$, we find

$$
I_{3} / I_{1}=-0.7014 \text {. }
$$

It can be seen that $I_{3} / I_{1}$ too is always negative. This can be explained by the presence of the factor $m_{2}^{2}$ in (26): heavy field stars have much more effect in encounters. Since $m_{1}+m_{2}$ appears in the denominator of the argument of the logarithm, the true mean value of the logarithm is less than the classical value, obtained by replacing $m_{1}$ and $m_{2}$ by the average $\langle m\rangle$.

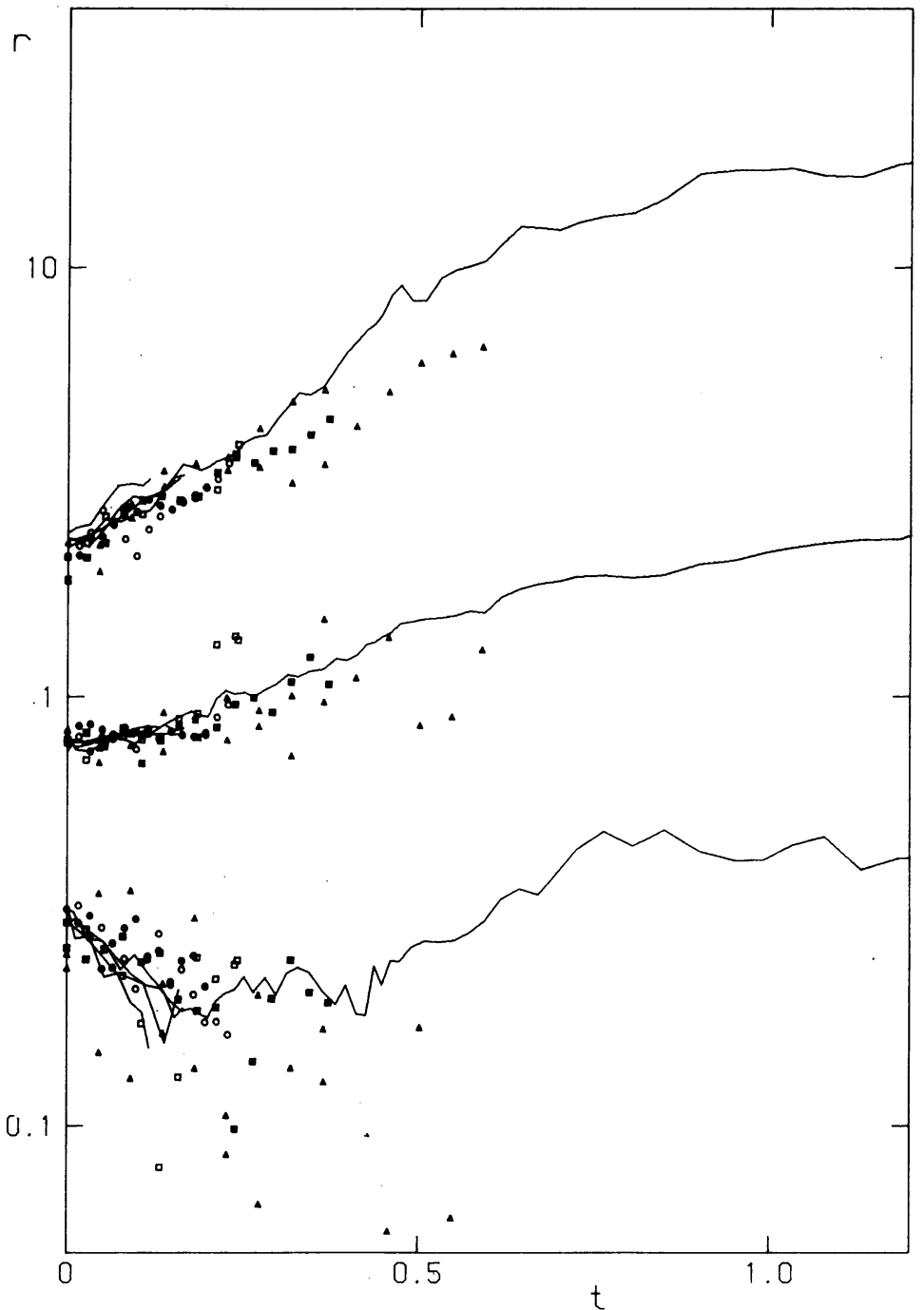

Fig. 6. Comparison of four Monte Carlo models (full lines) with six $N$-body simulations by Wielen (symbols) for the case of Wielen's mass distribution, using the new value $\gamma=0.075$. The Monte Carlo models are the same as in Figure 3. Triangles, squares and circles correspond to $N=100,250,500$ respectively. The initial state is the same as before. 
We note also that the corrective term $I_{3} / I_{1}$ becomes more and more important as the dispersion of the masses increases. Therefore one cannot meaningfully quote a mean value here. In view of our previous result (19), the value of $\gamma$ to be used should in general be

$$
\gamma=0.15 \exp \left(I_{3} / I_{1}\right),
$$

where $I_{3} / I_{1}$ depends on the particular mass distribution, and should be evaluated from (26) or from one of our Tables.

For Wielen's distribution, we derive from (33) and (34) the rounded value

$$
\gamma=0.075 \text {. }
$$

Figure 6 shows again the four Monte Carlo models of Figure 3 (full lines), together with the results of six $N$-body simulations by Wielen (1974) (filled and open symbols). The value $\gamma=0.075$ is used. This figure should be compared with Aarseth et al. (1975, Figure 2) or Wielen (1975, Figure 2), where the classical value $\gamma=0.4$ was used. Here again the agreement is much better with the new value, although the Monte Carlo models still appear to run somewhat too fast. The corrective factor (24), with $\gamma=0.4$ and $\gamma^{\prime}=0.075$, is $1.8308,1.5711,1.4619$ respectively for $N=100,250,500$, the values used in the $N$-body simulations of Figure 6. It is apparent here that the effect of the non-dominant terms is far from negligible.

The agreement found here, particularly in the case of equal masses (Figure 5), may be somewhat accidental, since there are many other approximations in the theory which we have not considered. For instance, we know that the largest impact distance should be of the order of the dimensions of the system; but its particular identification with the radius containing half the mass, in (3), is no more than a guess. If this maximum impact distance were halved or doubled, the constant $\gamma$ would also be halved or doubled.

Nevertheless, our results show at least that there exists no significant disagreement between the results of $N$-body simulations and Monte Carlo models. The previously noted differences (Aarseth et al., 1975) can be entirely accounted for by the approximations in the theory. Thus the classical theory, founded on the assumption that evolution is due to the cumulative effect of two-body encounters, appears to be confirmed by the results of $N$-body simulations.

\section{Acknowledgement}

We thank Dr Aarseth for helpful comments on this paper.

\section{References}

Aarseth, S. J.: 1971, Astrophys. Space Sci. 13, 324.

Aarseth, S. J.: 1973, Vistas in Astronomy 15, 13.

Aarseth, S. J.: 1974, Astron. Astrophys. 35, 237. 
Aarseth, S. J.: 1975, this volume, p. 57.

Aarseth, S. J., Hénon, M., and Wielen, R.: 1975, Astron. Astrophys. 37, 183.

Abramowitz, M. and Stegun, I. A.: 1965, Handbook of Mathematical Functions, Dover, New York, p. 1004 Chandrasekhar, S.: 1941, Astrophys. J. 93, 285.

Chandrasekhar, S.: 1942, Principles of Stellar Dynamics, Chicago University Press, Chicago.

Hénon, M.: 1961, Ann. Astrophys. 24, 369.

Hénon, M.: 1971, Astrophys. Space Sci. 13, 284 and 14, 151.

Hénon, M.: 1973, in L. Martinet and M. Mayor (eds.), Dynamical Structure and Evolution of Stellar Systems, Swiss Society of Astronomy and Astrophysics Third Advanced Course, Geneva Observatory, p. 224.

King, I.: 1975, this volume, p. 99.

Larson, R. B.: 1970, Monthly Notices Roy. Astron. Soc. 147, 323; $150,93$.

Liboff, R. L.: 1959, Phys. Fluids 2, 40.

Lynden-Bell, D. and Wood, R.: 1968, Monthly Notices Roy. Astron. Soc. 138, 495.

Spitzer, L., Jr.: 1969, Astrophys. J. 158, L139.

Spitzer, L., Jr.: 1975, this volume, p. 3.

Spitzer, L., Jr. and Hart, M. H.: 1971, Astrophys. J. 164, 399.

Spitzer, L., Jr. and Thuan, T. X.: 1972, Astrophys. J. 175, 31.

Von Hoerner, S.: 1968, Bull. Astron., Sér. 3, 3, 147.

Wielen, R.: 1967, Veröffentl. Astron. Rechen-Inst. Heidelberg, Nr. 19.

Wielen, R.: 1971, Astrophys. Space Sci. 13, 300.

Wielen, R.: 1974, in L. N. Mavridis (ed.), Proceedings of the First European Astronomical Meeting (Athens 1972), Springer-Verlag, Berlin-Heidelberg-New York, Vol. 2. p. 326.

Wielen, R.: 1975, this volume, p. 119.

\section{DISCUSSION}

Larson: Do I understand correctly that the time scale correction that you have derived applies equally to any theory based on the Fokker-Planck equation?

Hénon: Yes. In particular, this correction will also improve the agreement between your fluid-dynamical models and the $N$-body simulations. 